\title{
Análise Espacial Aplicada ao Estabelecimento de Novos Varejos Alimentícios na Cidade de Maringá/PR: Estudo de Caso de Geomarketing
}

\author{
Spatial Analysis Applied to the Establishment of new Food Retail in the \\ City of Maringá / PR: Geomarketing Case of Study \\ Rodrigo Blaudt Lima da Silva ${ }^{1}$ \\ Otávio Cristiano Montanher ${ }^{2}$
}

Recebido em maio de 2019. Aprovado em novembro de 2019.

\begin{abstract}
RESUMO
A crescente competitividade de mercado e a popularização dos Sistemas de Informação Geográfica têm levado a uma necessidade de integração de dados e metodologias para geração de análises espaciais robustas. Este trabalho objetiva apresentar um estudo de caso que busca mapear regiões potenciais para a instalação de varejos alimentícios no município de Maringá-PR. A partir de um conjunto de dados viários, locacionais e sociodemográficos gratuitos, foi gerado um mapa síntese através do método AHP em ambiente SIG, subsidiado por métodos de interpolação como krigagem ordinária e regressão polinomial. Em comparação com estudos de caráter qualitativo, o resultado da aplicação desta metodologia permitiu uma integração objetiva de diversas variáveis em um mapa síntese. O potencial de instalação de varejos alimentícios se concentrou no centro da cidade, outras duas manchas com alto potencial de instalação foram identificadas, uma no setor leste da cidade e a outra à noroeste. Ressalta-se que o mapeamento de síntese apresenta a variação espacial do potencial para instalação de novos varejos alimentícios, sem a especificação de qual tipo.
\end{abstract}

PALAVRAS-CHAVE: Geomarketing. Krigagem. AHP.

\begin{abstract}
The crescent competition in the retail business and the popularization of Geographic Information System has emerged the need for integration between data and methodologies for making robust spatial analyses. The present work aims to show a case study which persuades to map potential areas for food retail establishment in the city of Maringá-PR. From a free
\end{abstract}

\footnotetext{
${ }^{1}$ Universidade Estadual de Maringá. Graduação em Geografia, Brasil. E-mail: rodrigo.blaudt@outlook.com ${ }^{2}$ Universidade Estadual de Maringá. Departamento de Geografia, Brasil. E-mail:
} otaviocmontanher@yahoo.com.br 
available dataset that contains road lines, food retail location and sociodemographic, it has been generated a synthetic map by using AHP methodology in GIS software, based on interpolation methods like ordinary kriging and polynomial regression. In comparison with qualitative studies, the results of applying this methodology proved to be effective in allowing the integration of several variables into a single synthesis map. The potential areas for food retail establishment were concentrated in the center of the town, two other areas that show high potential location for installation, one in the eastern sector of the city and the other in the northwest. The syntheses mapping presents the spatial variability for potential areas for the installation of food retail, without specifying which type.

KEYWORDS: Geomarketing. Kriging. AHP

$$
* * *
$$

\section{Introdução}

Em decorrência da disponibilidade crescente de dados sociodemográficos e de infraestrutura urbana, novas soluções têm surgido para o planejamento, tanto em âmbito público como privado. Do contexto de popularização dos Sistemas de Informação Geográfica (SIGs) e da possibilidade de espacialização de dados surge o geomarketing, sendo o intermédio entre a geografia e o marketing, e a partir disto, aplicações relacionadas aos atributos espaciais dos agentes que constituem a relação comercial (ARANHA, 1996; GREGORI e LINK, 2006; FURLAN, 2011).

Apesar de Aranha (1996) alertar por futura maior aderência do Geomarketing devido à limitada solução de problemas apresentada pelos métodos tradicionais de marketing e Furlan (2011) constatar sua tendência de novidade e inovação, Serafim (2014) acusa a baixa quantidade de estudos na área. Entretanto, como Gregori e Link (2006) e Felini (2017) relatam, aplicações deste tipo de estudo por empresas são realizadas em quantidade substancial. Considerando também a cientifização dos negócios, a maior competitividade no setor comercial e a seletividade dos consumidores em relação ao produto e ao local de compra, torna-se fundamental para sobrevivência dos comércios a ampliação do uso de dados, bem como o 
desenvolvimento de técnicas e análises integradas (GREGORI e LINK, 2006; SERAFIM, 2014).

Dessa forma, destaca-se o recorrente uso de sistemas de informação para apoio à tomada de decisão na área de marketing. A integração do geomarketing a estes sistemas viabiliza uma série de aplicações, tais como: análise de potencial de mercado, segmentação de mercado, localização de clientes atuais e potenciais, auxílio no marketing-mix, análise demográfica em regiões especificas, campanhas de marketing direto, localização de concorrentes e geradores de fluxos, entre outros (GREGORI e LINK, 2006; MACHADO, FRANCISCO e RIBEIRO, 2006; SERAFIM, 2014).

Tendo como base tais potencialidades do geomarketing, este trabalho teve como objetivo apresentar um estudo de caso relacionado a identificação de áreas com potencial de instalação de novos varejos alimentícios na cidade de Maringá-PR. Procedimentos de interpolação de dados sociodemográficos (IBGE, 2010) por krigagem e regressão polinomial, bem como de ponderação das variáveis - sociodemográficas e estruturais - por meio do Processo Hierárquico Analítico (AHP) foram utilizados para compor o mapa síntese.

A escolha das variáveis sociodemográficas utilizadas neste trabalho são derivadas por um lado a partir de Felini (2017), que a através de análises de correlação linear verificou quais características socioeconômicas da população mais se relacionam à existência de varejos alimentícios no município de Maringá-PR. Por outro lado, através de Moura (2006), que obteve resultados por meio de entrevistas in loco, da segmentação do perfil socioeconômico dos consumidores dos diferentes tipos de varejo alimentício. A metodologia é foi desenvolvida a partir de Silva, Montanher e Silva (2018).

\section{2 Área de estudo}

O município de Maringá está localizado no norte central paranaense (Figura 1), e cujo planejamento foi elaborado pela Companhia Melhoramentos Norte do Paraná no final da década de 1940. Segundo o IBGE (2010), o Índice 
de Desenvolvimento Humano (IDHM) de Maringá em 2010 era de 0,808 superior ao IDHM do Brasil e do Paraná. Segundo o mesmo instituto, a população total era de 357.077 mil habitantes, em 2018 a população total foi estimada em 417.010 mil habitantes.

O município é um importante polo de desenvolvimento das atividades do setor têxtil, agroindustrial e comercial (RIPPEL e LIMA, 2009). Além de ter sido considerado em 2017 e 2018 como o melhor município do Brasil, dentre as 100 maiores cidade do país, para se viver (MACROPLAN, 2018).

Foram utilizados os setores censitários como unidade espacial de análise devido à sua escala intra-urbana. A área urbana do município de Maringá possui 541 setores censitários (excluindo os distritos), em que alguns polígonos que não contêm dados (como áreas verdes, por exemplo), foram excluídos. Ao todo, 521 setores foram utilizados (Figura 1). A Figura 1 também apresenta os centroides dos setores censitários, eles constituem feições pontuais, as quais foram utilizadas nos procedimentos de interpolação.

Figura 1 - Localização da área de estudo com detalhe para os setores censitários que cobrem a área urbana do município de Maringá e respectivos centroides

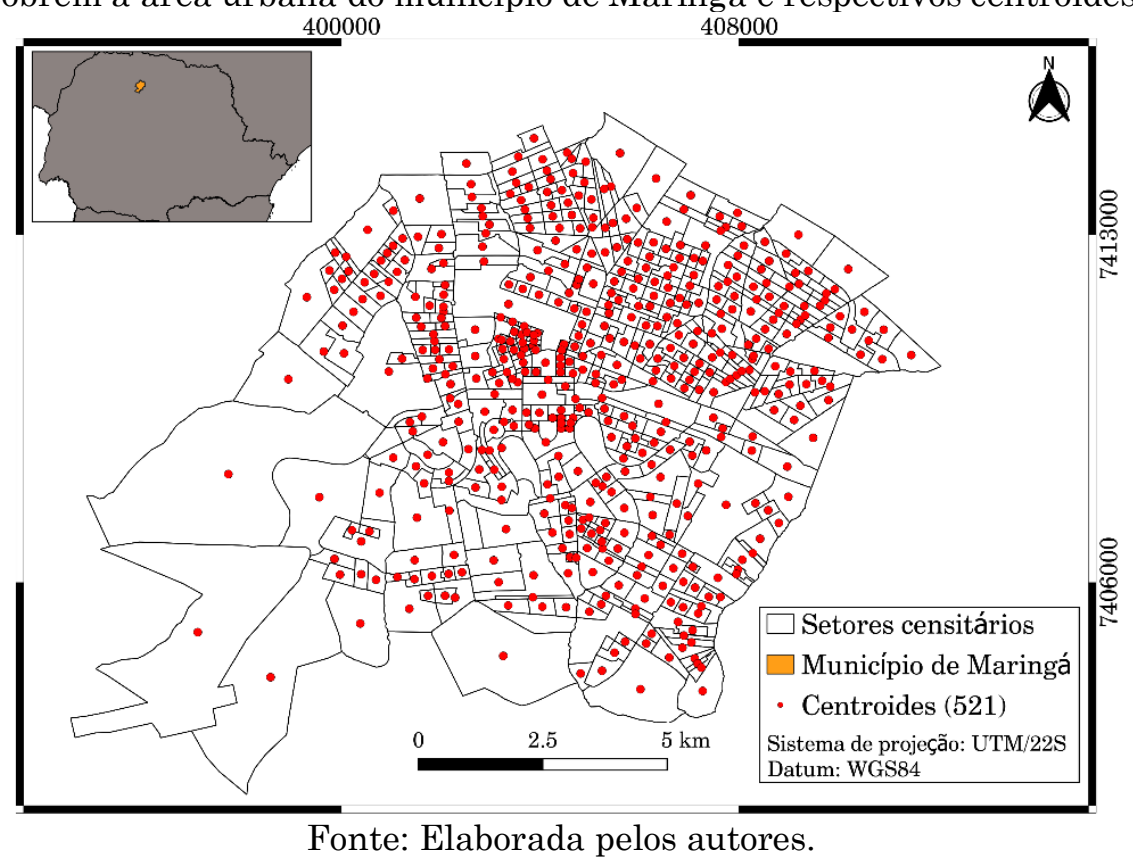




\section{Metodologia}

A metodologia do presente trabalho foi divida em três etapas: i) préprocessamento; ii) processamento; e iii) síntese (Figura 2). A primeira etapa consistiu na coleta dos dados estruturais e sociodemográficos - também denominados de categorias no decorrer do texto. A categoria estrutural contempla os dados de localização dos varejos alimentícios concorrentes e da estrutura viária hierarquizada do município. Como apresentado pela Figura 2, a categoria sociodemográfico se refere às variáveis censitárias (IBGE, 2010): i) total do rendimento nominal mensal dos domicílios particulares permanentes $(\mathrm{R})$; ii) moradores em domicílios particulares permanentes (M); e iii) razão entre o total de mulheres e homens em domicílios particulares permanentes $(\mathrm{H})$.

O processamento configura a segunda etapa da metodologia. Como apontado pela Figura 2, cada categoria (vias, concorrência e sociodemografia) foi subdividida em três variáveis: vias (primárias, secundárias e terciárias), concorrência (hipermercados, supermercados e minimercados) e sociodemografia ( $\mathrm{R}, \mathrm{M}$ e H). Ainda nesta etapa, os endereços dos concorrentes foram geocodificados e todas as variáveis foram transformadas em arquivos matriciais (do inglês raster). Para as variáveis da categoria estrutural (concorrentes e vias) a transformação ocorreu através da geração de raster de distância entre as variáveis de uma mesma categoria. As variáveis da categoria sociodemográfica foram transformadas através de técnicas de interpolação.

Como cada categoria e cada variável apresentam importâncias diferentes para a composição do mapa síntese, o método AHP foi utilizado para definição desses pesos em dois momentos: i) etapa de processamento cruzamento entre as variáveis intracategóricas; e ii) etapa de síntese ponderação intercategóricas (Figura 2). Este procedimento permitiu que a ponderação das variáveis fosse realizada tendo como base a revisão bibliográfica. 
$\mathrm{Na}$ etapa de processamento foi aplicado o método AHP em cada um desses grupos de variáveis, para que fossem ponderados e sintetizados em uma única camada raster. Ao final da etapa de processamento, obteve-se três raster, um para cada categoria. Na etapa de síntese, que consistiu na geração do mapa de potencial de instalação de varejos alimentícios, a técnica AHP foi executada entre as camadas raster geradas como resultado da etapa de processamento (Figura 2).

Todos os procedimentos deste trabalho foram executados com uso de softwares livres. Em relação à organização do banco de dados, conversões entre tipos de vetores e operações entre arquivos raster, foi utilizado o QGIS (QGIS DEVELOPMENT TEAM, 2009). Vetores de estrutura viária foram obtidos no portal Open Street Map (www.openstreetmap.org). Os endereços dos varejos alimentícios existentes no município foram geocodificados através da extensão MMQGIS (MINN, 2019). Para análise variográfica e ajuste de modelos espaciais de autocorrelação foi utilizado o pacote gstat (PEBESMA, 2019), operacional em ambiente R (R CORE TEAM, 2017). Após o ajuste dos variogramas experimentais, a krigagem foi executada no software SAGA (CONRAD et al., 2015). Para execução da Análise Hierárquica de Processos (AHP) utilizou-se a extensão Easy AHP (BILGIN, 2014), operacional em ambiente QGIS.

Os procedimentos metodológicos foram desenvolvidos a partir de Silva, Montanher e Silva (2018). O desenvolvimento ocorreu principalmente na forma como foi realizada a interpolação do conjunto de variáveis sociodemográficas e na distribuição dos pesos relativos para geração do mapa síntese (Figura 2).

Neste estudo a krigagem ordinária, utilizada para predição da superfície continua das variáveis $\mathrm{R}$ e $\mathrm{M}$ foi executada a partir da análise variográfica em ambiente $R$. A superfície continua da variável $H$ da categoria sociodemográfica, por não apresentar dependência espacial, deixando de atender aos pressupostos da krigagem, foi obtida através de regressão 
polinomial. A metodologia de interpolação aplicada aos dados sociodemográficos será detalhada na seção seguinte.

Figura 2 - Fluxograma metodológico

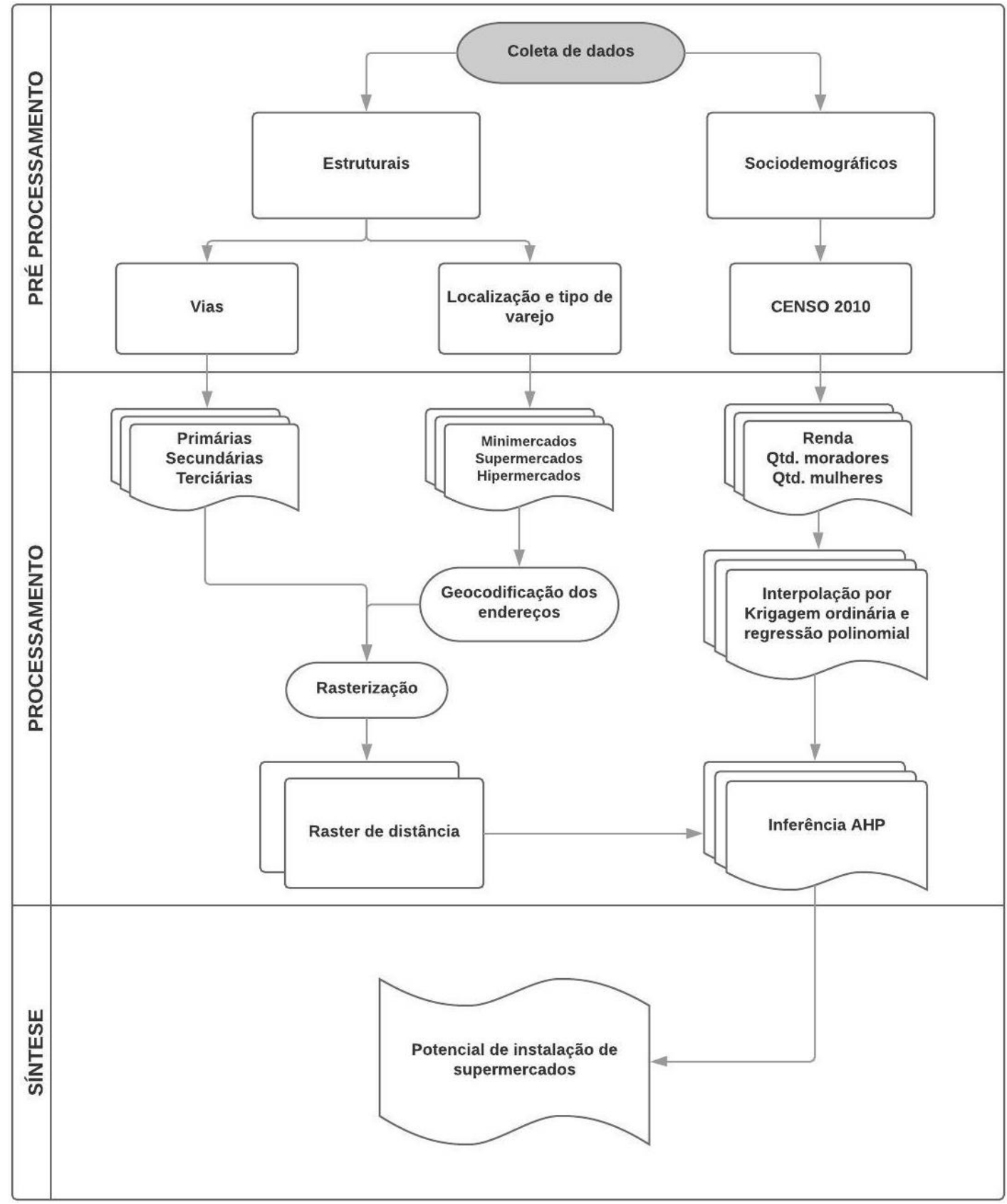

Fonte: Adaptada de Silva, Montanher e Silva (2018).

A categoria viária compreende as vias primárias, secundárias e terciárias, classificadas pelo portal Open Street Map (www.openstreetmap.org). Os varejos alimentícios foram classificados 
conforme a Classificação Nacional de Atividades Econômicas (CONCLA, 2019) em hipermercados, supermercados e minimercados, através de consultas do CNPJ obtido no portal da Secretaria da Fazenda do Paraná (http://www.fazenda.pr.gov.br), e geocodificados a partir do endereço.

A classificação utilizada (CONCLA, 2019) diferencia os varejos alimentícios conforme tipos, baseado nos produtos vendidos e área de venda. Os minimercados são definidos por área de venda inferior a $300 \mathrm{~m}^{2}$, os supermercados apresentam área de venda entre 300 e $5.000 \mathrm{~m}^{2}$ e os hipermercados área de venda superior a $5.000 \mathrm{~m}^{2}$.

Para operacionalização entre as categorias selecionadas, optou-se pela utilização de dados raster obtidos por interpoladores espaciais, tais como a krigagem ordinária e a regressão polinomial. Como recomendado por Sampaio (2012), o resultado de cada saída raster foi normalizado entre 0 e 1 para posterior geração do mapa síntese ponderado pelo método AHP.

\subsection{Interpolação de variáveis sociodemográficas}

Os dados dos setores censitários representam os levantamentos referentes a cada setor (feição poligonal), sem qualquer diferenciação interna a cada unidade espacial. Para obtenção de superfícies que apresentassem variação contínua a partir dessa base de dados, foram extraídos os centroides de cada setor (feições pontuais), os quais mantiveram a tabela de atributos do vetor poligonal. Posteriormente, os pontos foram utilizados em procedimentos de interpolação. Existe uma ampla gama de interpoladores, em que uma diferenciação se baseia no uso de modelos determinísticos locais, globais, ou modelos estatísticos locais e globais (DRUCK et al., 2004). A última classe mencionada de interpoladores utiliza princípios geoestatísticos, os quais funcionam com base no conhecimento da dependência espacial dos valores de uma determinada variável aleatória, enquanto os interpoladores determinísticos utilizam funções matemáticas sem este princípio (DRUCK et al., 2004; YAMAMOTO e LANDIM, 2013). 
Para avaliação da autocorrelação espacial dos dados, os variogramas das três variáveis utilizadas nesta pesquisa foram avaliados. No entanto, a análise geoestatística demanda uma série de pressupostos. Um desses pressupostos se refere à distribuição da variável Z. A krigagem ordinária pode ser aplicada diretamente em dados com distribuição normal ou com assimetria negativa, ao passo que variáveis que possuam assimetria positiva (como uma distribuição lognormal) devem ser transformadas (YAMAMOTO e LANDIM, 2013). A distribuição das variáveis sociodemográficas foi avaliada por meio de seus histogramas.

Foi necessário aplicar a transformação para as variáveis renda e quantidade de moradores, enquanto a variável razão entre homens e mulheres apresentou uma boa aproximação para uma distribuição normal sem qualquer modificação. A transformação por logaritmo natural fez com que os dados apresentassem forte assimetria negativa, por isso optou-se pela transformação via raiz quadrada (Figura 3).

Após a transformação, para avaliação do pressuposto de estacionaridade (DRUCK et al., 2004), avaliou-se a distribuição dos dados conforme as coordenadas UTM N e E (eixos X e Y). Observou-se que haviam tendências a serem removidas anteriormente à análise via variograma. Todas as três variáveis foram ajustadas por meio de regressão polinomial, em que foram adotados polinômios de terceira ordem. Após a geração da superfície de tendência, foram obtidos os resíduos por subtração simples.

Com base na análise dos variogramas dos resíduos (Figura 4) observouse que as variáveis $\mathrm{R}$ e M possuem dependência espacial, sendo estas tratadas por meio de krigagem ordinária, enquanto a variável H não apresentou. Como essa última variável não passou por transformação e não apresentou dependência espacial, sua superfície contínua foi obtida diretamente por meio do interpolador de regressão polinomial dos dados originais. Pelo fato de a razão entre homens e mulheres $(\mathrm{H})$ não ter apresentado forte variabilidade e um padrão relativamente simples, com maiores valores na região central e 
menores nas bordas da área urbana, julgou-se adequado esse modelo determinístico de efeito global.

Figura 3 - Histogramas das três variáveis sociodemográficas (as variáveis renda e quantidade de moradores passaram pela transformação por raiz quadrada, enquanto a variável razão entre homens e mulheres não apresenta fortes desvios em relação à distribuição normal)
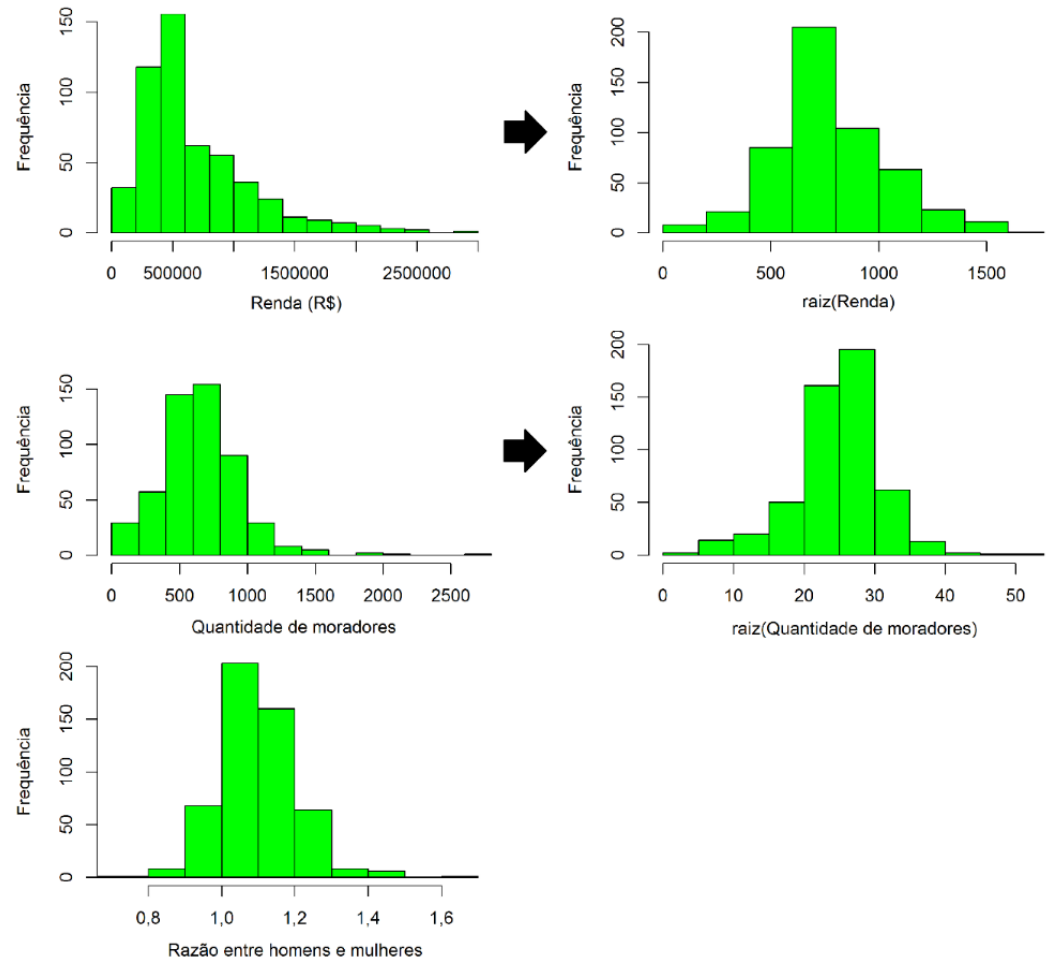

Fonte: Elaborada pelos autores.

Figura 4 - Variogramas das variáveis sociodemográficas: renda (A), quantidade de moradores (B) e razão entre homens e mulheres (C) (as curvas apresentadas em A e B referem-se aos modelos exponenciais ajustados)
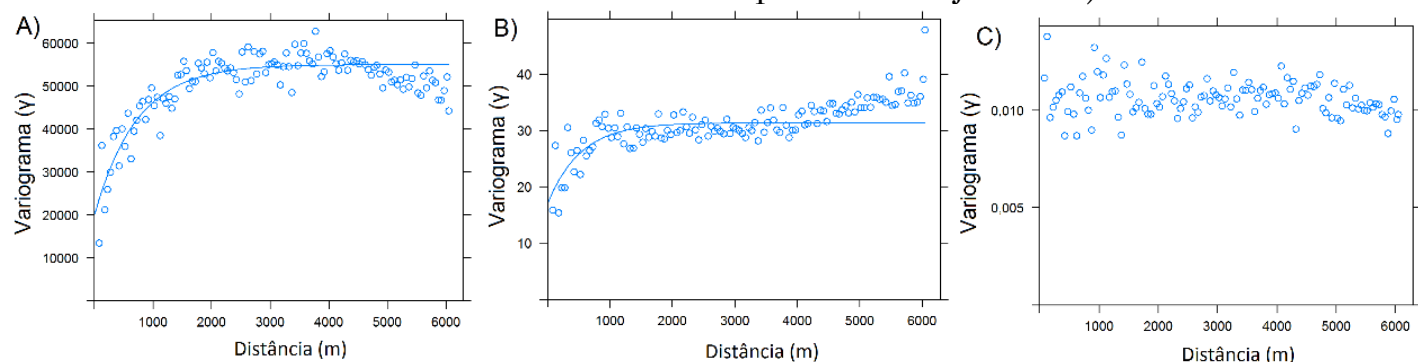

Fonte: Elaborada pelos autores.

Adicionalmente, avaliou-se o pressuposto de isotropia das variáveis $R$ e M por meio de variogramas direcionais, em quatro direções: $0^{\circ}, 45^{\circ}, 90^{\circ} \mathrm{e}$ $135^{\circ}$ (YAMAMOTO e LANDIM, 2013). Os variogramas não apresentaram 
fortes padrões anisotrópicos (Figura 5), embora para a variável renda na direção de $0^{\circ}$ haja uma leve diminuição da semivariância após $4.000 \mathrm{~m}$ de distância. Isso deve explicar o comportamento do variograma geral desta variável, com diminuição da semivariância a partir da mesma distância (Figura 4).

Figura 5 - Variogramas direcionais das variáveis renda (A) e quantidade de moradores (B)
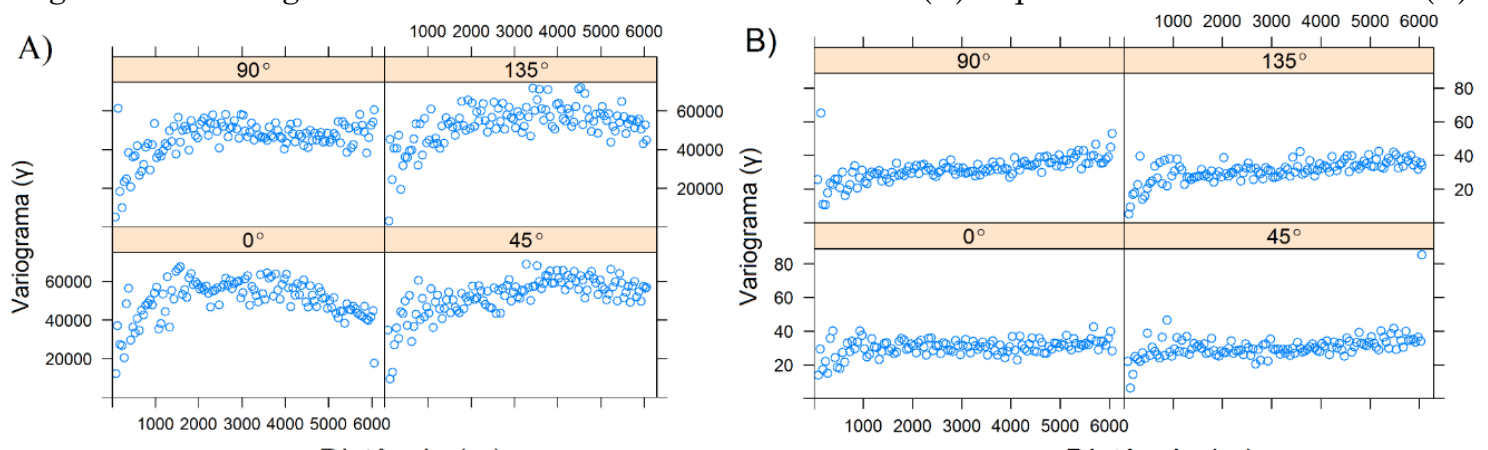

Fonte: Elaborada pelos autores.

No entanto, considerou-se que tais efeitos não necessitavam de tratamento, pois, após a distância de $4.000 \mathrm{~m}$ o número de pares utilizados para o cálculo da semivariância diminui consideravelmente. Uma mudança de forma da função variograma também pode ser observada na variável quantidade de moradores (Figura 4B), com aumento da semivariância a partir de $4.000 \mathrm{~m}$. Portanto, os modelos foram ajustados com as informações obtidas até a distância de $4.000 \mathrm{~m}$.

Tabela 1 - Descrição dos modelos ajustados às variáveis renda $(R)$ e quantidade de moradores $(\mathrm{M})$. A variável h refere-se à distância

\begin{tabular}{c|c|c|c|c|c}
\hline Variável & Modelo & Alcance & Pepita & Patamar & Equação ajustada \\
\hline$\sqrt{R}$ & \multirow{2}{*}{ Exponencial } & 722 & 19.967 & 55.011 & $\begin{array}{r}19.967+35.044 \cdot(1 \\
\left.-\mathrm{e}^{-\frac{h}{722}}\right)\end{array}$ \\
\cline { 4 - 6 } & & 528 & 17 & 31,4 & $17+14.4 \cdot\left(1-\mathrm{e}^{-\frac{h}{528}}\right)$ \\
\hline
\end{tabular}

Fonte: Elaborada pelos autores.

Levando em conta uma boa aproximação ao atendimento dos pressupostos geoestatísticos, a krigagem foi aplicada às variáveis $\mathrm{R} e \mathrm{M}$, cujos modelos estão descritos na Tabela 1. A forma dos modelos pode ser observada nas curvas ajustadas na Figura 4. Os arquivos raster resultantes da krigagem 
foram somados às superfícies de tendência (polinômios de terceira ordem) e o último passo foi a transformada inversa (Figura 6).

Figura 6 - Camadas raster interpoladas por krigagem das variáveis $\mathrm{R}$ e M

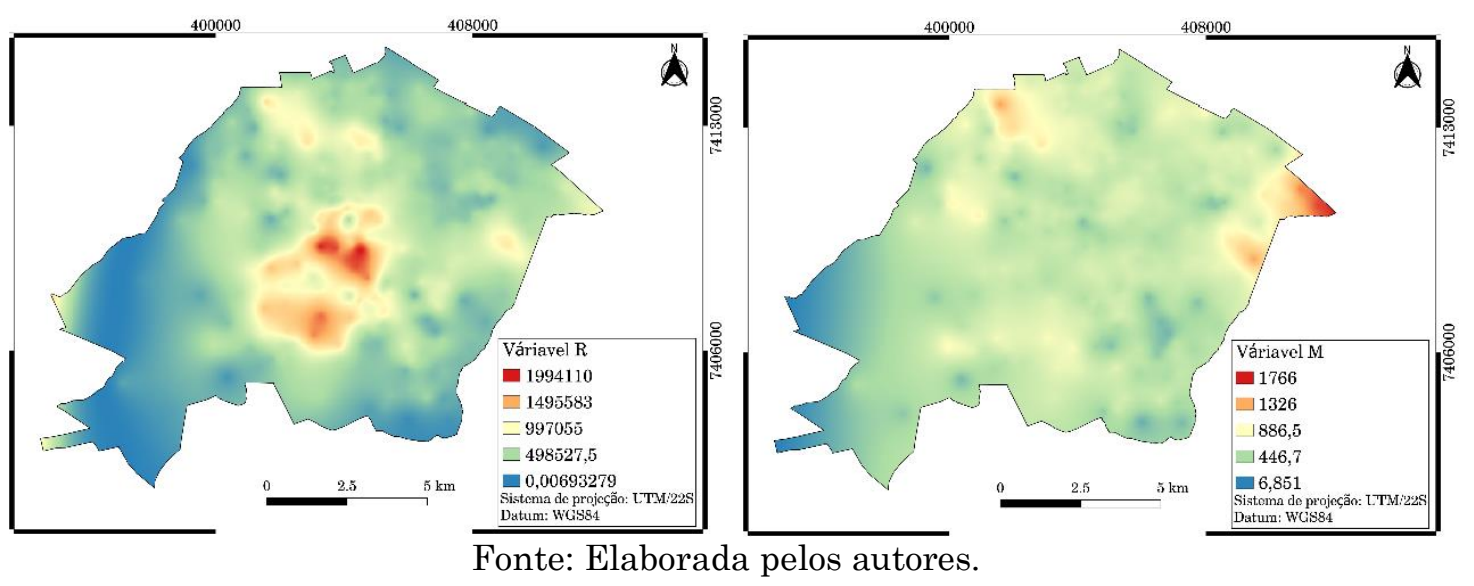

\section{Resultados e discussão}

Apesar de o procedimento de krigagem gerar uma superfície estimada e suavizada do valor dos dados interpolados (JAKOB, 2016), o resultado desta aplicação gera uma superfície matricial que possibilita operações entre diferentes variáveis consideradas para a análise (CÂMARA et al., 2001). No caso deste estudo a operacionalização das variáveis e suas respectivas categorias foram realizadas através do método AHP. O peso das variáveis calculado através do método AHP para cada categoria é apresentado na Tabela 2 e as superfícies geradas a partir desta ponderação nas Figuras 7, 8 e 9. O resultado dos pesos para o mapa síntese calculado entre as categorias encontra-se na Tabela 3, o mapa síntese é apresentado na Figura 10.

Entre as categorias selecionadas para o estudo, a de maior relevância para elaboração do mapa síntese é a sociodemográfica, seguido por eixos viários e concorrência. Felini (2017) encontra correlação significativa entre dados sociodemográficos e a existência de varejos alimentícios, destacando renda e quantidade de moradores por domicilio. Moura (2006) também observou essa relação por meio de entrevistas in loco, porém, este ainda relata 
maior frequência de compras em supermercados das mulheres em relação aos homens.

Tabela 2 - Pesos atribuídos entre as categorias de variáveis pelo método AHP

\begin{tabular}{c|c|c}
\hline Categoria & Variável & Peso \\
\hline \multirow{3}{*}{ Sociodemografia } & $\mathrm{R}$ & 0,458 \\
\cline { 2 - 3 } & $\mathrm{M}$ & 0,416 \\
\cline { 2 - 3 } & $\mathrm{H}$ & 0,126 \\
\hline \multirow{3}{*}{ Eixos Viários } & Primário & 0,623 \\
\cline { 2 - 3 } & Secundário & 0,239 \\
\cline { 2 - 3 } & Terciário & 0,137 \\
\hline \multirow{3}{*}{ Concorrência } & Hipermercados & 0,122 \\
\cline { 2 - 3 } & Supermercados & 0,648 \\
\cline { 2 - 3 } & Minimercados & 0,23 \\
\hline
\end{tabular}

Fonte: Elaborada pelos autores.

Os pesos AHP conferidos à categoria viária (Figura 8) consideram maior relevância às vias primárias, seguido pelas secundárias e terciárias. Grassi (2010) em análise qualitativa de locais para expansão de supermercados, seleciona grandes avenidas como fator chave à sua instalação. Dentre as variáveis da categoria concorrência, o maior peso foi conferido aos supermercados, hipermercados e minimercados, respectivamente.

Tabela 3 - Atribuição dos pesos as categorias para elaboração do mapa síntese

\begin{tabular}{c|c}
\hline Categoria & Peso \\
\hline Sociodemografia & 0,581 \\
\hline Eixos viários & 0,309 \\
\hline Concorrência & 0,110 \\
\hline
\end{tabular}

Fonte: Elaborada pelos autores.

Apesar de Parente e Kato (2001) apresentam em um estudo da área de abrangência de cinco varejos alimentícios em São Paulo, optou-se por não estabelecer uma delimitação espacial da área de abrangência para cada tipo de varejo alimentício de Maringá, com base nos resultados do estudo citado, como fez Grassi (2010). Isto ocorreu pois é esperado que cada cidade seja caracterizada por dinâmicas de consumo singulares em determinado tempo- 
espaço, o que implica na impossibilidade de generalizar modelos gerados em lugares e tempos tão distintos.

Figura 7 - Superfície gerada pelo método AHP para a categoria sociodemografia

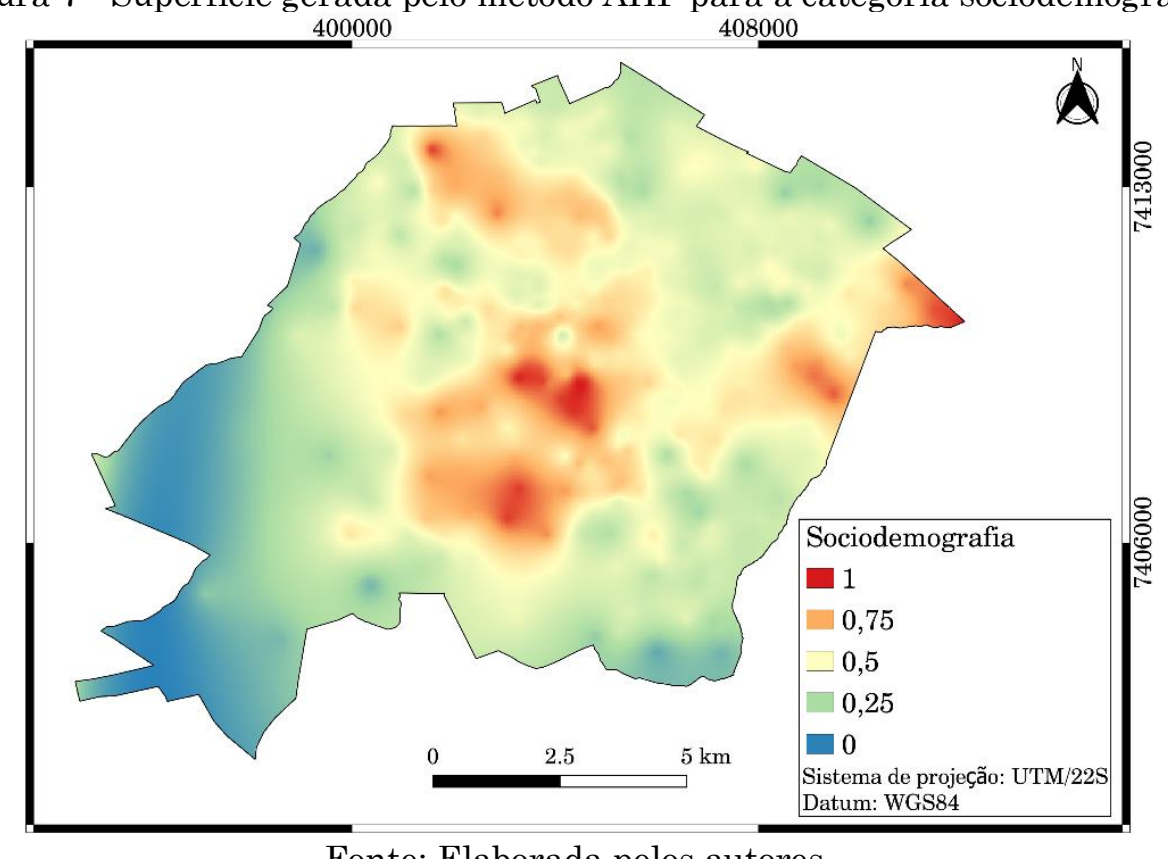

Fonte: Elaborada pelos autores.

Gregori e Link (2006) com semelhante objetivo, desenvolvem modelos a partir da correlação entre variáveis sociodemográficas e o faturamento por setor censitário de determinada franquia. Como geralmente o faturamento é um dado de acesso limitado, para garantir maior aplicação e acessibilidade do geomarketing, a metodologia proposta no presente trabalho se mostra possível em qualquer situação.

Apesar da utilização de dados censitários de 2010, Santos (1996, apud Felini, 2017), considera que a estrutura espacial se altera em ritmo lento, mantendo uma configuração territorial constante, apesar da possível alteração dos valores dos dados. Esta característica permite a utilização de dados coletados em 2010 para geração de mapa síntese em 2019. Apesar disso, o ideal é o uso de dados atualizados. 
Figura 8: Superfície gerada pelo método AHP para a categoria vias

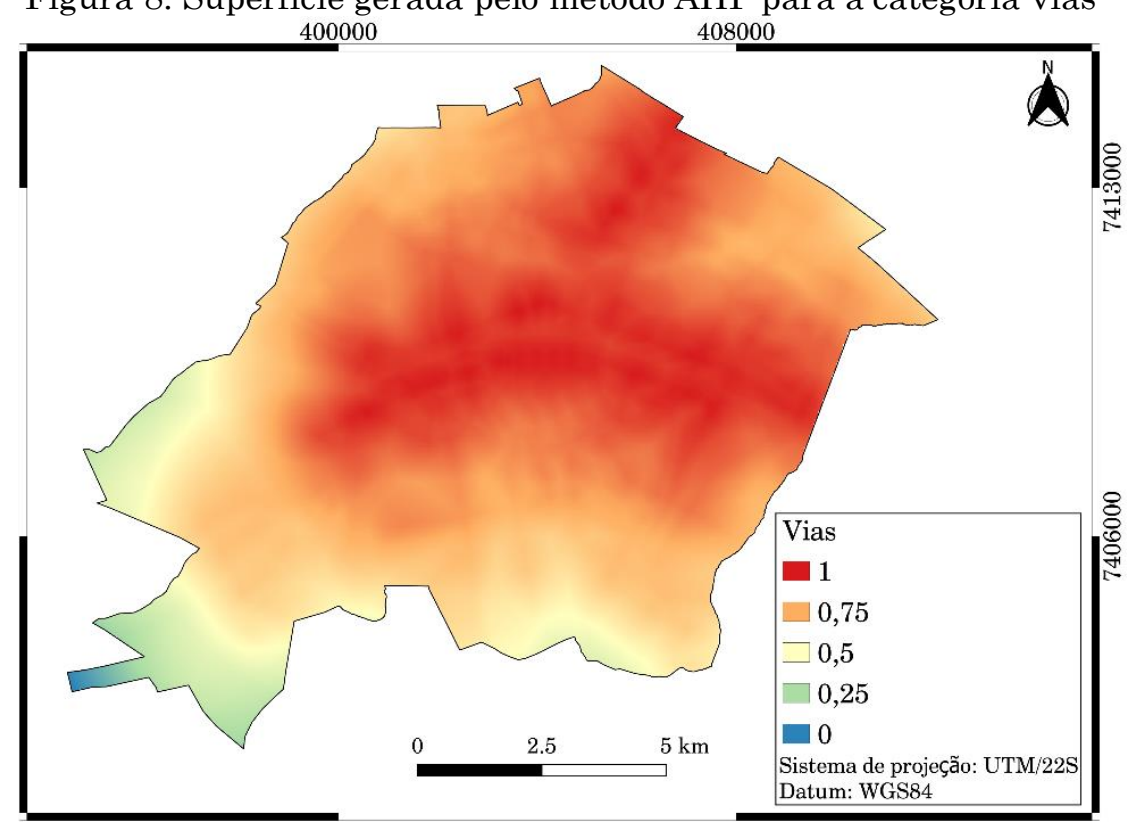

Fonte: Elaborada pelos autores.

Ainda que Grassi (2010) tenha obtido relativo sucesso ao propor locais para instalação de um novo supermercado em Porto Alegre/RS, a partir da análise qualitativa dos dados, questiona-se se foi possível contemplar na análise todas as áreas da cidade. A partir da metodologia quantitativa do presente trabalho, o resultado gerado considera todas as variáveis inseridas na delimitação espacial de interesse (Figura 9). Dessa forma, há ganhos consideráveis de tempo e certeza de que o resultado apresentado abrange a análise de todas as variáveis, por toda a área de estudo.

Além disso, como a metodologia empregada para atingir tal objetivo depende apenas da entrada dos pesos relativos atribuídos no método AHP, faz-se ainda possível, utilizando o mesmo banco de dados, a geração de produtos para instalação de minimercados ou hipermercados através da atribuição de diferentes pesos às variáveis/categorias. Essas alterações estariam baseadas em literatura já constatadas em Moura (2006), ou através de uma análise espacial mais detalhada, a partir de Felini (2017). Outra forma possível seria através de questionários aplicados in situ, que poderia complementar a síntese através da informação da localização do consumidor com a simples questão: "Costuma vir de casa ou do trabalho para o 
supermercado?", aproximando-se do estudo realizado por Gregori e Link (2006), que considera a população trabalhadora (diurna) e a população residente.

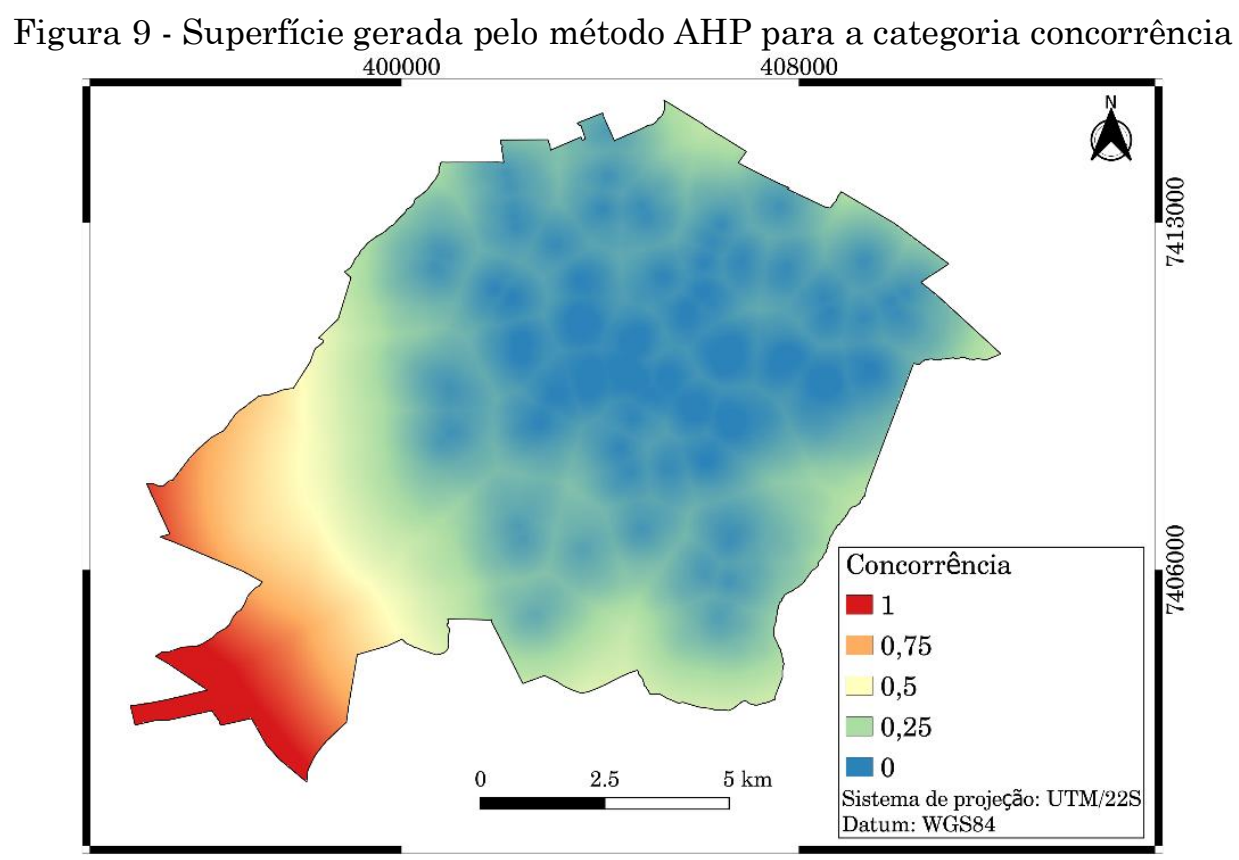

Fonte: Elaborada pelos autores.

É importante frisar que os resultados das análises espaciais apresentadas neste artigo referem-se aos dados obtidos por meio do censo de 2010, ou seja, encontram-se desatualizados. Um dos problemas decorrentes disso é que a atual análise desconsidera novos bairros que surgiram no período. Ao se observar a Figura 10, pode-se notar que alguns bairros não foram incluídos na área de estudo, justamente por serem bairros que surgiram após o último censo demográfico. A única ressalva é para a área localizada a leste da área de estudo, cujos bairros pertencem a outro município (Sarandi).

Uma sugestão para análises futuras é que a metodologia apresentada neste artigo seja aplicada para os dados do próximo censo. Isso permitiria uma avaliação atualizada da disposição espacial da potencialidade para instalação de novos mercados, bem como a avaliação desse potencial em regiões da cidade que não foram incluídas nesta pesquisa. Por último, ainda 
seria recomendada a comparação, onde possível, das variáveis-síntese de 2010 e do próximo censo, o que permitiria a observação de possíveis tendências espaciais de mudanças no perfil consumidor em escala intraurbana. O noroeste do município também é apontado como uma região de alto potencial para instalação de varejos alimentícios.

Figura 10 - Mapa síntese do potencial de instalação de varejo alimentício 400000 408000

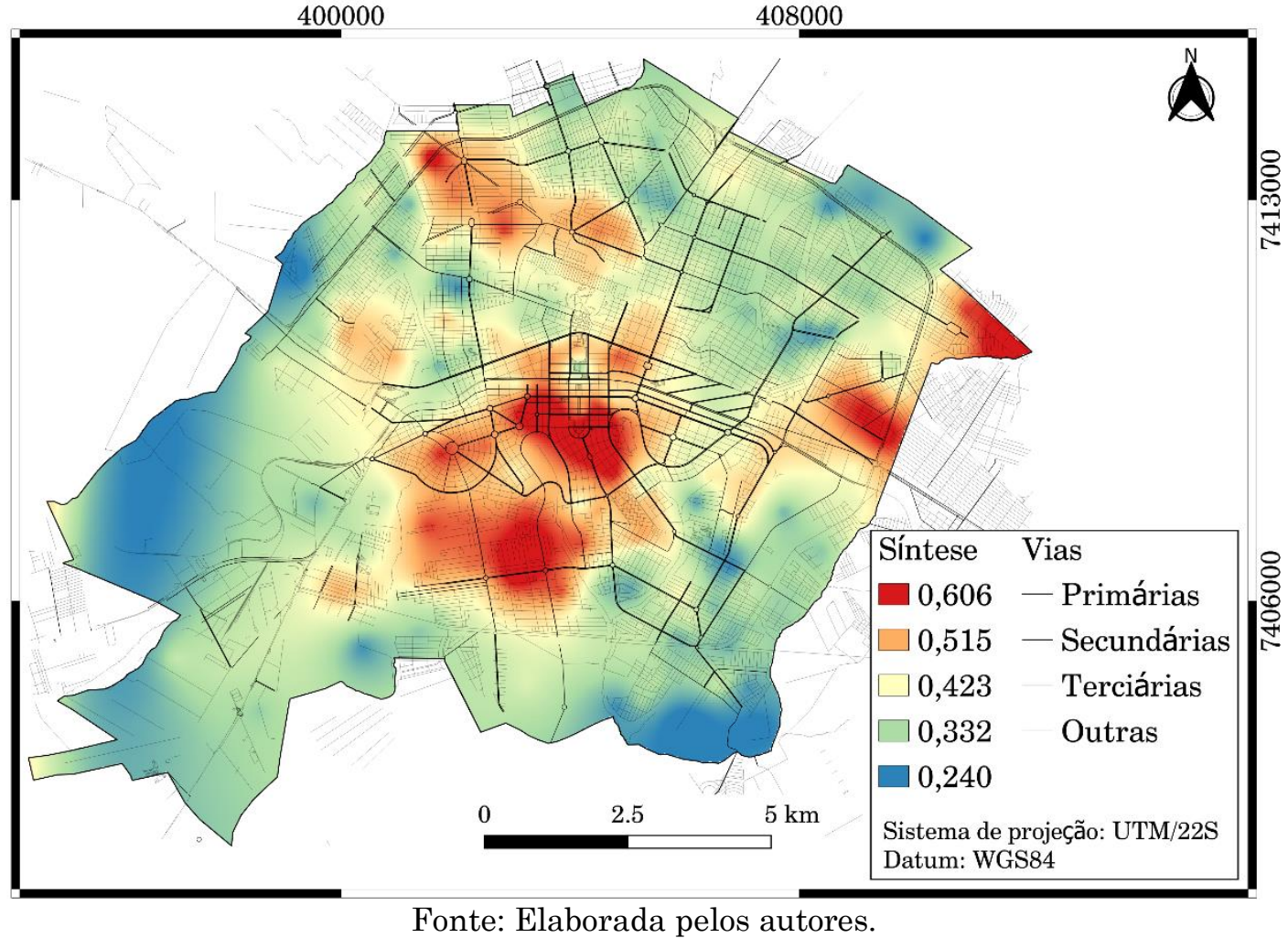

\section{Conclusões}

O mapeamento de síntese permite inferir que o potencial para instalação de novos varejos alimentícios se concentra na região central da cidade dado a relevância da variável renda, observado tanto através do método da interpolação por krigagem como na realidade urbana.

Além da distribuição com sentido centro-periferia, outras duas manchas de alto potencial para instalação foram identificadas, as quais diferem desse padrão espacial geral da cidade. Uma no setor leste da cidade, 
onde a quantidade de moradores é alta e o fator de concorrência é baixo. E a outra no setor noroeste, onde renda e quantidade de moradores são expressivas. Outras variações espaciais menores existem, mas com menor intensidade.

Por fim, é importante frisar que o mapeamento de síntese apresenta a variação espacial do potencial para instalação de novos varejos alimentícios, sem a especificação de qual tipo. As características sociodemográficas de cada bairro, bem como o custo do aluguel/aquisição do imóvel, irão determinar quais tipos de varejo atendem a demanda local.

\section{Agradecimentos}

Os autores gostariam de agradecer ao Programa de Iniciação Científica da Universidade Estadual de Maringá.

\section{Contribuição dos autores}

Rodrigo Blaudt Lima da Silva foi responsável pela conceptualização, investigação, curadoria dos dados, metodologia, visualização e redação. Otávio Cristiano Montanher foi responsável pela análise formal, administração do projeto, supervisão, validação e revisão da redação. 


\section{Referências}

ARANHA, F. Sistemas de Informação Geográfica: Uma Arma Estratégica para a Database Marketing. Revista de Administração de Empresas (FGV). São Paulo, v.36, n.2, 1996. pp.12-16.

BILGIN, M. S. QGIS Python Plugins Repository: Easy AHP. Site $<$ https://plugins.qgis.org/plugins/EasyAHP/>. Acessado em dezembro de 2018.

CÂMARA, G.; DAVIS, C.; MONTEIRO, A.M.; D’ALGE, J.C. Introdução à ciência da geoinformação. São José dos Campos, INPE, 2001.

CONCLA, Comissão Nacional de Classificação. Tabela CNAE, 2019. Site $<$ http://www.cnae.ibge.gov.br>. Acessado em: fevereiro de 2019.

CONRAD, O.; BECHTEL, B.; BOCK, M.; DIETRICH, H.; FISCHER, E.; GERLITZ, L.; WEHBERG, J.; WICHMANN, V.; BÖHNER, J. System for Automated. Geoscientific Analyses (SAGA) v. 2.1.4. Geoscientific Model Development, vol. 8, 2015. pp. 1991-2007.

DRUCK, S.; CARAVALHO, M.S.; CÂMARA, G.; MONTEIRO, A.V.M. Análise Espacial de Dados Geográficos. Brasília, EMBRAPA, 2004.

FELINI, M. G. Geomarketing: o caso dos supermercados de Maringá - PR. 2017. Trabalho de Conclusão de Curso. Bacharelado em Geografia. Universidade Estadual de Maringá, Departamento de Geografia, Maringá, 2017. 62p.

FURLAN, A. A. Geoprocessamento: estudos de Geomarketing e as possibilidades de sua aplicação no planejamento do desenvolvimento socioeconômico. Revista GEOUSP: Espaço e Tempo. São Paulo, n. 29, 2011. pp. 97-105.

GRASSI, V. Estratégias de localização de uma rede de supermercados: o geomarketing aplicado à companhia Zaffari em porto alegre - RS. Trabalho de Conclusão de Curso. Bacharelado em Geografia. Universidade Federal do Rio grande do Sul, Rio Grande do Sul, 2010. 52p.

GREGORI, R. G.; LINK, F. A cientifização dos negócios: visão geral e estudo de caso do setor financeiro. In: GUIMARÃES, J. R. S. Demografia dos negócios: campo de estudo, perspectivas e aplicações. Campinas: ABEP, 2006. pp. 97-130. 
HASHIMOTO, F. H. N.; NETO, O. C. P. O Geomarketing como Instrumento para a Pesquisa de Mercado: Estudo de caso do Supermercado Sacolão Big Dog em Londrina-PR. Geografia. Londrina, vol.28, n.1, 2019. pp.133-149.

IBGE, Instituto Brasileiro de Geografia e Estatística. Censo Demográfico 2010. Site $<$ https://censo2010.ibge.gov.br>. Acessado em: janeiro de 2019.

JAKOB, A.A.E. A Krigagem como método de análise de dados demográficos. Anais do encontro nacional de estudos populacionais, Minas Gerais, 2002.

MACHADO, C. C.; FRANCISCO, E. de R.; RIBEIRO, J. G. P. A Geodemografia e o geomarketing na identificação de mercados potenciais. Demographicas, 2006. pp. 77-95.

MACROPLAN. Desafios da Gestão Municipal, 2018. Site $<$ https://www.macroplan.com.br>. Acessado em dezembro de 2018.

MINN, M. Describes use of MMQGIS, a set of Python vector map layer plugins for Quantum GIS. Site <http://michaelminn.com/linux/mmqgis/>. Acessado em janeiro de 2019.

MOURA, T. L.; SILVA, A. L.; BATALHA, M. O. Perfil dos consumidores que frequentam os formatos de varejo de alimentos no Brasil. Anais do Encontro da ANPAD, Salvador, 2006.

PARENTE, J.; KATO, H. T. Área de influência: um estudo no varejo de supermercados. Revista de Administração de Empresas (FGV). São Paulo, v.41, n.2, 2001. pp. 46-53.

PEBESMA E. Gstat User's Manual. Site <http://www.gstat.org/manual/>. Acessado em janeiro de 2019.

QGIS DEVELOPMENT TEAM. QGIS Geographic Information System. Open Source Geospatial Foundation. Site <https://www.qgis.org/pt_BR/site/>. Acessado em abril de 2019 .

R CORE TEAM. R: A language and environment for statistical computing. $\mathrm{R}$ Foundation for Statistical Computing, Vienna, Austria. 2017. Site $<$ https://www.R-project.org/>. Acessado em janeiro de 2019.

RIPPEL, R.; LIMA, J.F. Polos de crescimento econômico: notas sobre o caso do estado do Paraná. Revista Redes. Santa Cruz do Sul, n. 1, v. 14, 2009. pp. 136-149. 
SAMPAIO, T. V. M. Diretrizes e procedimentos metodológicos para a cartografia de síntese com atributos quantitativos via álgebra de mapas e análise multicritério. Boletim de Geografia, Maringá, v. 30, n. 1, 2012. pp. 121-131.

SANTOS, M. A natureza do espaço. São Paulo: Hucitec, v. 1, 1996. 384p.

SERAFIM, V. F. M. Sistemas de informação de marketing, geomarketing e níveis estratégicos em organizações no Brasil. Dissertação de mestrado. Universidade Nova de Lisboa, Instituto Superior de Estatística e Gestão de Informação, Lisboa, 2014. 191p.

SILVA, R. B. L.; MONTANHER, O. C.; SILVA, J. P.; Geomarketing para supermercados Maringá/PR. Anais do IV Encontro Regional de Geografia e XXVI Semana de Geografia, Paraná, 2018. pp. 65-77.

YAMAMOTO, J. K.; LANDIM, P. M. B. Geoestatística conceitos e aplicações. São Paulo, Editora Oficina de Textos, 2013. 216p. 